J. Austral. Math. Soc. 25 (Series A) (1978), 118-128

\title{
ON THE STRUCTURE AND EXISTENCE OF SOME AMICABLE ORTHOGONAL DESIGNS
}

\author{
PETER J. ROBINSON and JENNIFER SEBERRY \\ (Received 30 March 1977; revised 23 June 1977) \\ Communicated by W. D. Wallis
}

\begin{abstract}
The structure is determined for the existence of some amicable weighing matrices. This is then used to prove the existence and non-existence of some amicable orthogonal designs in powers of two.
\end{abstract}

Subject classification (Amer. Math. Soc. (MOS) 1970): 05B20, 15A36, $62 \mathrm{~K} 99$.

\section{Introduction}

An orthogonal design of order $n$ and type $\left(u_{1}, u_{2}, \ldots, u_{s}\right)\left(u_{i}>0\right)$ on the commuting variables $x_{1}, x_{2}, \ldots, x_{s}$ is an $n \times n$ matrix $A$ with entries from $\left\{0, \pm x_{1}, \ldots, \pm x_{8}\right\}$ such that

$$
A A^{\mathrm{T}}=\sum_{i=1}^{s}\left(u_{i} x_{i}^{2}\right) I_{n}
$$

Alternatively, the rows of $A$ are formally orthogonal and each row has precisely $u_{i}$ entries of type $\pm x_{i}$.

In Geramita et al. (1975-76), where this was first defined and many examples and properties of such designs were investigated, it is mentioned that

$$
A^{\mathbf{T}} A=\sum_{i=1}^{s}\left(u_{i} x_{i}^{2}\right) I_{n},
$$

and so the alternative description of $A$ applies as well to the columns of $A$. It is also shown in the same paper that $s \leqslant \rho(n)$, where $\rho(n)$ (Radon's function) is defined by

$$
\rho(n)=8 c+2^{d},
$$

when $n=2^{a} . b, b$ odd, $a=4 c+d, 0 \leqslant d<4$. In Wolfe (1975) it is shown that if $n \equiv 4(\bmod 8)$ and if there exists an orthogonal design of order $n$ and type $\left(a_{1}, a_{2}, a_{3}, a_{4}\right)$ then $\Pi_{i<j}\left(a_{i}, a_{j}\right)_{p}=1$ at all primes $p$ where $\left(a_{i}, a_{j}\right)_{p}$ is the Hilbert norm residue symbol. D. Shapiro (private communication, 1975-76) has found conditions on possible $\rho(n)$-tuples which can be the types of orthogonal designs in all orders $n$. In Geramita and Verner (1976) it is observed that if there exists an 
orthogonal design of type $\left(u_{1}, u_{2}, \ldots, u_{l}\right)$ in order $n \equiv 0(\bmod 4)$ with $\sum_{i=1}^{t} u_{i}=n-1$ there exists a design of type $\left(1, u_{1}, u_{2}, \ldots, u_{1}\right)$ in order $n$. Robinson (1976b) has shown that orthogonal designs of type $(1,1,1,1,1, n-5)$ cannot exist in orders $n>40$.

Wolfe (1976) defines two orthogonal designs $X, Y$, of the same order and types $\left(x_{1}, \ldots, x_{8}\right)$ and $\left(y_{1}, \ldots, y_{t}\right)$ respectively satisfying

$$
X Y^{\mathbf{T}}=Y X^{\mathbf{T}},
$$

to be amicable orthogonal designs of types $\left(\left(x_{1}, \ldots, x_{s}\right) ;\left(y_{1}, \ldots, y_{t}\right)\right)$. He gives conditions on the number of variables in such designs. Such amicable orthogonal designs were first used (in another guise) to construct Hadamard matrices. Some are given in Geramita et al. (1975-76) and some infinite families constructed in Wallis (1975).

A weighing matrix of weight $k$ and order $n$ is a square $\{0,1,-1\}$ matrix, $W=W(n, k)$, or order $n$ satisfying

$$
W W^{\mathbf{T}}=k I_{n} \text {. }
$$

In Geramita et al. (1975-76) it is shown that the existence of an orthogonal design of order $n$ and type $\left(u_{1}, \ldots, u_{s}\right)$ is equivalent to the existence of weighing matrices $A_{1}, \ldots, A_{g}$, of order $n$, where $A_{i}$ has weight $u_{i}$ and the matrices, $\left\{A_{i}\right\}_{i=1}^{g}$, satisfying the matrix equation

in pairs.

$$
X Y^{\mathbf{T}}+Y X^{\mathbf{T}}=0
$$

\section{Preliminaries}

Let

$$
\begin{gathered}
K=\left[\begin{array}{rr}
0 & 1 \\
-1 & 0
\end{array}\right], \quad L=\left[\begin{array}{rr}
1 & 0 \\
0 & -1
\end{array}\right], \quad M=\left[\begin{array}{ll}
0 & 1 \\
1 & 0
\end{array}\right], \\
I=\left[\begin{array}{ll}
1 & 0 \\
0 & 1
\end{array}\right] \text { and } H=\left[\begin{array}{rr}
1 & 1 \\
1 & -1
\end{array}\right]
\end{gathered}
$$

We shall write + for +1 and - for -1 . Also we use $I$ for the identity matrix and $\oplus_{k} Z$ for the direct sum of $k$ copies of $Z$.

Matrices $A, B$ which satisfy $A B^{\mathrm{T}}=B A^{\mathrm{T}}$ will be said to be amicable. A best pair is a pair of amicable weighing matrices $(A, B)$ of weights $i, j$ respectively satisfying

$$
A^{\mathrm{T}}=-A, \quad B^{\mathrm{T}}=B, \quad A B^{\mathrm{T}}=B A^{\mathrm{T}} .
$$

A best pair family of order $n$ is a set of best pairs $\left(A_{i}, B_{j}\right)$ of order $n$ and weights $i$ and $j$ where

and

$$
i=1,2,3, \ldots, n-1, \quad j=1,2,3, \ldots, n
$$

$$
A_{i}^{\mathrm{T}}=-A_{i}, \quad B_{j}^{\mathrm{T}}=B_{j}, \quad A_{i} B_{j}^{\mathrm{T}}=B_{j} A_{i}^{\mathrm{T}} .
$$


Let $R, P_{1}, P_{2}, \ldots, H$ be orthogonal designs of order $n$ and types $\left(r_{1}, r_{2}, \ldots\right)$, $\left(p_{i 1}, p_{i 2}, \ldots\right)$ for $i=1,2, \ldots,\left(h_{1}, h_{2}, \ldots\right)$ respectively. Then $\left(R ;\left(P_{1} ; P_{2} ; \ldots\right) ; H\right)$ are repeat designs of order $n$ and types $\left(r_{1}, r_{2}, \ldots ;\left(p_{11}, p_{12}, \ldots ; p_{21}, p_{22}, \ldots ; \ldots\right) ; h_{1}, h_{2}, \ldots\right)$

(i) $R * P_{i}=0$ for $i=1,2, \ldots$ (* the Hadamard product);

(ii) $R+P_{i}$ for $i=1,2, \ldots$ are orthogonal designs;

(iii) $R+P_{i}$ and $H$ for $i=1,2, \ldots$ are amicable orthogonal designs;

(iv) $P_{i} P_{j}^{\mathrm{T}}=P_{j} P_{i}^{\mathrm{T}}$ for $i \neq j$.

Repeat designs are defined and used extensively in Robinson and Seberry (to appear) to study orthogonal designs. For convenience we will call a repeat design $(P ;(R ; S) ; \mathrm{H})$ of type $(1 ;(r ; s) ; h)$ in order $n$ a triplet when $P=I$. Alternatively, a triplet is three weighing matrices $(R, S, H)$ of order $n$ and weights $(r, s, h)$ respectively which are pairwise amicable; $R, S$ are skew symmetric and $H$ is symmetric. We note that

LEMMA 1. There are triplets in orders $n=2$ and 4 for weights $(i, j, k)$ where $i, j=1,2, \ldots, n-1$ and $k=1,2, \ldots, n$. Hence, there is a best pair family of orders 2 and 4.

Proof. For order 2 consider the pairs $(K, M)$ and $(K, H)$. The required matrices for order 4 are (the weights are given in brackets):

1. $(1,2,1)\{K \times I, K \times H ; L \times I\}$,

2. $(1,2,2)\{K \times I, K \times H ; L \times H\}$,

3. $(1,2,3)\{K \times I, K \times H ; L \times H+M \times I\}$,

4. $(1,2,4)\{K \times I, K \times H ; H \times H\}$,

5. $(1,3,1)\{K \times L, K \times I+H \times K ; M \oplus-L\}$,

6. $(1,3,2)\{K \times L, L \times I+H \times K ; H \times L\}$,

7. $(1,3,3)\{K \times L, K \times I+H \times K ; M \oplus K+I \times M+K \times K\}$,

8. $(1,3,4)\{K \times L, L \times I+H \times K ; L \times I+M+L+I \times M+K \times K\}$,

9. $(2,3,1)\{I \times L+L \times M, L \times I+H \times L ; L \oplus-M\}$,

10. $(2,3,2)\{K+H, L \times K+M \times K+K \times I ; M \times I-L \times I\}$,

11. $(2,3,3)\{I \times L+L \times M, L \times I+H \times L ; M \oplus L+I \times M+L \times L\}$,

12. $(2,3,4)\{I \times K+K \times M, K \times I+H \times K ; L \times I+M \times L+I \times M+K \times K\}$.

Because of the extremely powerful constructions that arise from repeat designs we wished to extend this lemma to higher powers of two. This effort led to the results that follow. First, we give two useful lemmas and note a useful lemma of one of us (Robinson).

LEMMA 2. Suppose that $X$ and $Y$ are amicable orthogonal designs in order $n$, where $X$ is of type $\left(1, u_{1}, \ldots, u_{8}\right)$ on the variables $x_{0}, \ldots, x_{8}$ and $Y$ is of type $\left(v_{1}, \ldots, v_{l}\right)$ on the variables $y_{1}, \ldots, y_{i}$ then there exist monomial matrices $P$ and $Q$ (that is, with elements $0, \pm 1$ and only one non-zero element per row and column) so that

$$
P X Q=x_{0} I+\sum x_{i} M_{i}, \quad P Y Q=\Sigma y_{i} N_{i},
$$


where the $M_{i}$ 's and $N_{i}$ 's satisfy

(0) $M_{i} * M_{j}=0, i \neq j, N_{l} * N_{k}=0, l \neq k$;

(i) $M_{i}^{\mathrm{T}}=-M_{i} \forall i, N_{j}^{\mathrm{T}}=N_{j} \forall j$;

(ii) $M_{i} M_{j}^{T}=u_{i} I_{n}, N_{j} N_{j}^{\mathrm{T}}=v_{j} I_{n}$;

(iii) $M_{i} N_{j}^{\mathrm{T}}=N_{j} M_{i}^{\mathrm{T}}$;

(iv) $M_{i} M_{j}^{\mathrm{T}}+M_{j} M_{i}^{\mathrm{T}}=0, i \neq j, N_{l} N_{k}^{\mathrm{T}}+N_{k} N_{l}^{\mathrm{T}}=0, l \neq k$.

Proof. Choose $P$ and $Q$ so that the variable $x_{0}$ occurs on the diagonal of $P X Q$ and the rest follows immediately.

Construction 3. If $A, B, C$ is a triplet of weights $(a, b, 1)$ in order $n$, then $(A, B, A C)$ and $(A, B, A C+C)$ are triplets of weights $(a, b, a)$ and $(a, b, a+1)$.

Since by a lemma of P. J. Robinson (1976a) there is no best pair of weights $(7,5)$ in order 8 we have, regarding a best pair $(A, B)$ as a triplet $(X, A, B)$ :

COROLLARY 4. There are no triplets of weight $(x, 7,5),(4,7,1)$ or $(5,7,1)$ in order 8 .

In fact in order 8 we can say

LeMMA 5. In order 8 all triplets $(R, S, H)$ of weights $(r, s, h), 0 \leqslant r, s \leqslant 7,0 \leqslant k \leqslant 8$ exist except

(i) $(3,7,1),(4,7,1),(5,7,1),(6,7,1)$ and $(r, 7,5) 1 \leqslant r \leqslant 6$ which do not exist; and possibly

(ii) $(5,7,2),(6,7,2),(6,7,4),(3,7,3),(4,7,3),(5,6,3),(5,7,3),(6,7,3),(1,5,7)$, $(3,5,7),(3,7,7),(4,7,7),(5,7,7),(6,7,7)$ which are undecided.

Proof. Part (i) follows from the previous corollary and Theorem 15 which shows that $(3,7,1)$ and $(6,7,1)$ do not exist. Lemma 12(i), (iii), (iv), (v) of Robinson and Seberry (to appear) and the above construction give all those that exist except $(3,5,1),(3,5,5),(1,5,1),(1,7,1),(2,7,1)$ and $(1,7,3)$. Now we note that a repeat design of type $\left(r ;\left(p_{1}, \ldots ; q\right) ; w_{1}, \ldots\right)$ in order $n$ gives a repeat design of type $\left(r ;\left(q ; p_{1}, \ldots, q\right) ; w_{1}, \ldots\right)$ in order $2 n$ by Lemma 12 of Robinson and Seberry (to appear). Hence the repeat design of type $(1 ;(2 ; 3) ; 1)$ in order 4 gives the $(1 ;(3 ; 5) ; 1)$ in order 8 and hence the triplet $(3,5,1)$ and by Construction 3 the $(3,5,5)$ in 8 . We now give specific constructions for $(1,5,1),(1,7,1),(2,7,1)$ and $(1,7,3)$.

Let

$$
S=\left[\begin{array}{llll}
0 & 1 & 0 & 0 \\
0 & 0 & 1 & 0 \\
0 & 0 & 0 & 1 \\
- & 0 & 0 & 0
\end{array}\right], \quad R=\left[\begin{array}{llll}
0 & 0 & 0 & 1 \\
0 & 0 & 1 & 0 \\
0 & 1 & 0 & 0 \\
1 & 0 & 0 & 0
\end{array}\right], R^{2}=I
$$


and $K, L, M$ be as in Section 2. Write $E=-S+S^{2}+S^{3}$ and $G=S+S^{2}+S^{3}$. Then

and

$$
\left(L \times S^{2}, I_{2} \times\left(S+S^{3}\right)+K \times E R, L \times S^{2}\right)
$$

$$
\left(L \times S^{2}, L \times G+K \times(E+I)^{\mathrm{T}} R,\left(S^{3}+S-I\right)^{\mathrm{T}} R \oplus\left(-S^{2}-S+I\right)^{\mathrm{T}} R\right)
$$

have weights $(1,5,1)$ and $(1,7,3)$ respectively. Amnon Neeman found the following $(1,7,1)$ :

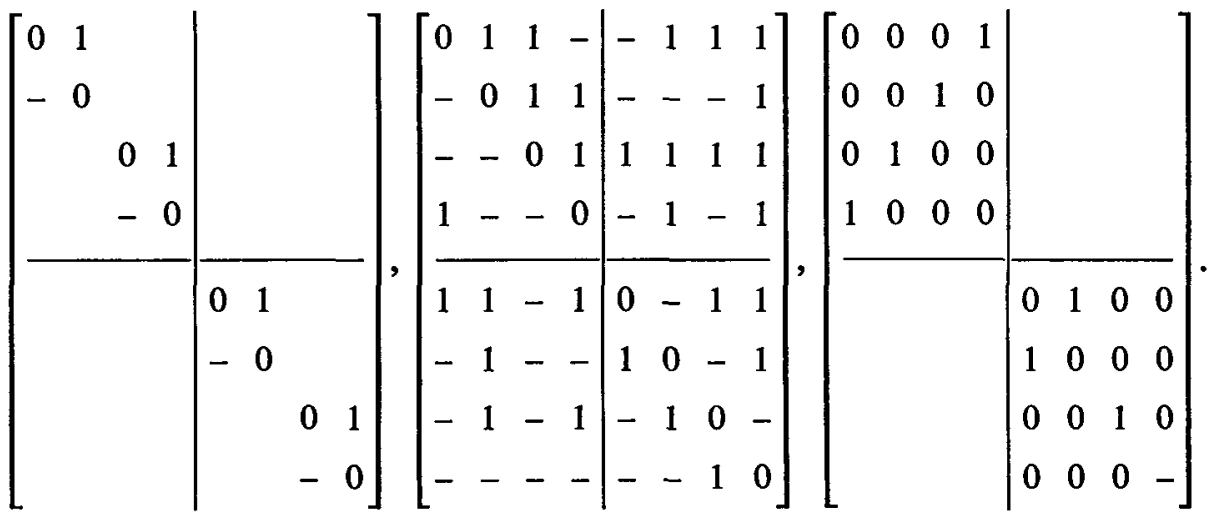

The following three matrices give a $(2,7,1)$ :

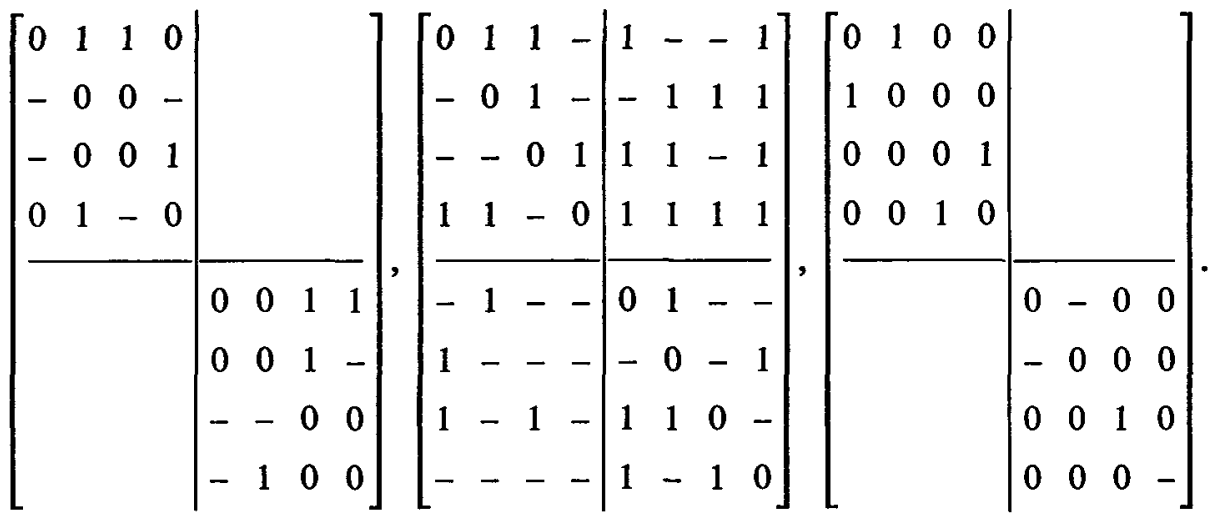

This gives the results of the enunciation after using Construction 3 to obtain $(1,5,5),(1,7,7)$ and $(2,7,7)$.

REMARK. This lemma indicates that the existence problem for triplets $(R, S, H)$ which are repeat designs $(I ;(R ; S) ; H)$ is very difficult and far from resolved.

But this lemma does allow us to say:

COROLlaRy 6. There are best pairs for all $(a, b), 0 \leqslant a \leqslant 7,0 \leqslant b \leqslant 8$ in order 8 except $(7,5)$. There are amicable weighing matrices for all $(a, b), 0 \leqslant a, b \leqslant 8$ in order 8. 
The results on repeat designs in Robinson and Seberry (to appear) allow us to say:

THeOREM 7. There are amicable weighing matrices for all $(a, b), 0 \leqslant a, b \leqslant 2^{t}$.

\section{The structure of amicable weighing matrices}

A combinatorial argument lets us obtain the following result.

THEOREM 8. Let $R$ be a monomial matrix of order $n \equiv 0(\bmod 4)$. Let $A$ be a symmetric weighing matrix of order $n$. Suppose that $R A^{\mathrm{T}}=-A R^{\mathrm{T}}$. Then $A * R=0$. Further, if $A$ has weight $n-1$ then $R$ is symmetric if $A$ has any diagonal element zero and $R$ is skew-symmetric otherwise.

THEOREM 9. The existence of amicable orthogonal designs of order $n \equiv 0(\bmod 4)$ and types $((1, n-1) ;(1, n-1))$ is equivalent to the existence of a symmetric weighing matrix of order $n$ and weight $n-1$ with at least one zero on the diagonal.

ProOf. Let $A$ be the symmetric weighing matrix. Use the theorem of Delsarte et al. (1971) to see that we can find monomial matrices $P$ and $Q$ so that $B=P A Q$ is skew-symmetric. Let $R=P^{-1} Q^{-1}$. Then $R$ is a monomial matrix and $B^{\mathrm{T}}=(P A Q)^{\mathrm{T}}=Q^{\mathrm{T}} A P^{\mathrm{T}}=Q^{-1} A P^{-1}=-P A Q$ so $R A^{\mathrm{T}}=-A R^{\mathrm{T}}$. Hence by the previous theorem $A * R=0$ and $R$ is symmetric. So $u R+v A$ and $x I+y A R$ are the required amicable orthogonal designs.

Now if $x U+y V$ and $u N+v M$ are amicable orthogonal designs of order $n$ and types $((1, n-1) ;(1, n-1))$. We pre and postmultiply both matrices by monomial matrices $P$ and $Q$ where $I=P U Q$. Then the amicable matrices can be written in the form $x I+y P V Q=x I+y S$ and $u P N Q+v P M Q=u R+v A$. Now the amicability and orthogonality gives us $R$ and $A$ are symmetric and $A R^{\mathrm{T}}=-R A^{\mathrm{T}}$. We now assume $A$ has weight $n-1$ and no zero on the diagonal. Then considering the orthogonality conditions on the rows of $A+R$ leads to a contradiction and we have the result.

REMARK. We note that this proof also shows that the existence of a symmetric weighing matrix $C$ of order $n$ and weight $n-1$ with a zero on the diagonal is equivalent to the existence of a best pair of weights $(n-1,1)$ in order $n$.

We recall (from Wallis, 1975) that amicable orthogonal designs of types $((1, p) ;(1, p))$ do exist in orders $p+1$ when $p \equiv 3(\bmod 4)$ is a prime power. Hence we have

COROLLARY 10. There is a symmetric weighing matrix with a zero on the diagonal of order $p+1$ and weight $p$ where $p \equiv 3(\bmod 4)$ is a prime power. 
We now observe that symmetric weighing matrices of order $n$ and weight $n-1$ with a zero on the diagonal do not always exist. We first observe:

LEMMA 11. If $(A, B)$ is $a(n-1,1)$ best pair in order $n \equiv 0(\bmod 4)$ then we may assume

$$
B=X \oplus\left[\begin{array}{cc}
\oplus & Y \\
\frac{1}{2} n-1 &
\end{array}\right]
$$

where

$$
X=\left[\begin{array}{rr}
-1 \\
0 & 1
\end{array}\right] \text { and } Y=\left[\begin{array}{ll}
0 & 1 \\
1 & 0
\end{array}\right]
$$

Proof. Firstly, we note that if $(A, B)$ is a best pair and $P$ is any permutation matrix then $\left(P A P^{\mathrm{T}}, P B P^{\mathrm{T}}\right)$ is a best pair of the same type.

It is obvious that we can find a $P$ such that

$$
P B P^{\mathrm{T}}=\underset{1 \leqslant i \leqslant i n}{\oplus} X_{i}
$$

where $X_{i}=X$ for $1 \leqslant i \leqslant k$ and $X_{i}=Y$ for $k+1 \leqslant i \leqslant \frac{1}{2} n$.

Since the top left-hand corner of $A$ is $\pm\left[\begin{array}{ll}0 & 1 \\ -0\end{array}\right] A B$ could not be symmetric if $X_{1}= \pm I_{2}$. So $X_{i}$ must be $X$ or $Y$ for all $i$.

Assume now that $X_{i}=Y$ for $1 \leqslant i \leqslant \frac{1}{2} n$ and

$$
A=\left[\begin{array}{ccccc}
0 & 1 & & & \\
- & 0 & A_{1} & A_{2} & \cdots \\
A_{1}^{\mathrm{T}} & & & \\
A_{2}^{\mathrm{T}} & & Z & \\
\vdots & & & &
\end{array}\right]
$$

where $A_{i}$ are $(2 \times 2)$ matrices with entries \pm 1 .

Let

Therefore

$$
A_{1}=\left[\begin{array}{ll}
x_{1} & x_{2} \\
x_{3} & x_{4}
\end{array}\right]
$$

$$
A_{1} Y=\left[\begin{array}{ll}
x_{2} & x_{1} \\
x_{4} & x_{3}
\end{array}\right] \text { and } A_{1}^{\mathrm{T}} Y=\left[\begin{array}{cc}
\bar{x}_{3} & \bar{x}_{1} \\
\bar{x}_{4} & \bar{x}_{2}
\end{array}\right] \text {. }
$$

Because $A B$ is symmetric, we must have $x_{2}=-x_{3}$ and $x_{1}=-x_{4}$, that is,

$$
A_{1}= \pm\left[\begin{array}{cc}
1 & 1 \\
- & -
\end{array}\right] \text { or } \pm\left[\begin{array}{ll}
1 & - \\
1 & -
\end{array}\right]
$$


This reasoning is also true for the other $A_{i}$ 's.

Now $\sum_{i=1}^{n-1} A_{i} A_{i}^{T}=(n-2) I_{n}$ by the orthogonality of $A$, and

$$
A_{i} A_{i}^{\mathrm{T}}=\left[\begin{array}{cc}
2 & -2 \\
-2 & 2
\end{array}\right] \text { or }\left[\begin{array}{ll}
2 & 2 \\
2 & 2
\end{array}\right] \text {. }
$$

But since there is an odd number of $A_{i}$ 's

$$
\sum A_{i} A_{i}^{\mathrm{T}} \neq(n-2) \text {. }
$$

Therefore at least one of the $X_{i}$ 's is $X$.

We now assume $X_{1}= \pm X_{2}=X$. Then we see the product $A B$ is not symmetric.

Therefore

$$
X_{1}=X \text { and } X_{i}=Y \text { for } 2 \leqslant i \leqslant \frac{1}{2} n .
$$

Hence we may assume $B$ is of the form given in (1).

We use this lemma to show that symmetric weighing matrices of order $n$ and weight $n-1$ with a zero on the diagonal do not always exist since

THEOREM 12. There is no best pair of weights $(15,1)$ in order 16 . Equivalently there is no symmetric weighing matrix of order 16 and weight 15 with a zero on the diagonal.

Proof. The proof is long and combinatorial. It is given in detail in Robinson (1977b).

\section{Some non-existence results}

Lemma 11 can be used to produce a result similar to a theorem of Wolfe (1976a):

THEOREM 13. There are no amicable orthogonal designs of order $n \equiv 0(\bmod 8)$ and types ((1); $(1, a, n-a-1)), a=2,3,4$ or 5 .

Proof. We assume that the matrix of weight 1 is of the form given by the lemma. A long, careful combinatorial argument gives the result. A fuller proof is given in Robinson (1977b).

COROLlary 14. There are no amicable orthogonal designs of order $n \equiv 0(\bmod 4)$ and types ((1); $(1, a, b, c)), a+b+c=n-1, a, b, c \neq 0$, and $a b c$ odd.

Proor. In the proof of the theorem we can see that each variable appears an even number of times off the diagonal $2 \times 2$ block and therefore one of $a, b$ and $c$ is odd.

We are grateful to Amnon Neeman for the proof of another result using Lemma 11: 
THEOREM 15. Let $n \equiv 0(\bmod 8)$. Then it is not possible to have triplets of weights $(a, n-1,1)$ in order $n$ where $a=n-4, n-3, n-2$, nor of weights $(3,7,1)$ in order 8 .

Proof. Lemma 11 allows us to consider the triplet $(X, Y, Z)$ where

$$
Z=\left[\begin{array}{c}
\oplus \\
\frac{1}{2} n-1
\end{array}\left[\begin{array}{ll}
0 & 1 \\
1 & 0
\end{array}\right]\right] \oplus\left[\begin{array}{cc}
- & 0 \\
0 & 1
\end{array}\right] .
$$

It is now possible, since $X Z^{\mathrm{T}}=Z X^{\mathrm{T}}$ and $X^{\mathrm{T}}=-X$, to decide the structure of the $2 \times 2$ submatrices of $X$. A careful analysis of the necessary structure for $X$ in each case of the enunciation shows it is not possible.

\section{Some existence results}

We take this opportunity to obtain some amicable orthogonal designs and a few repeat designs.

Consider:

$$
\begin{array}{rlrl}
\mathbf{X}_{1} & =\left[\begin{array}{rrrr}
x_{1} & x_{2} & x_{3} & x_{3} \\
-x_{2} & x_{1} & x_{3} & -x_{3} \\
x_{3} & x_{3} & -x_{1} & -x_{2} \\
x_{3} & -x_{3} & x_{2} & -x_{1}
\end{array}\right], & \mathbf{Y}_{1} & =\left[\begin{array}{rrrr}
y_{1} & y_{2} & y_{3} & y_{3} \\
y_{2} & -y_{1} & y_{3} & -y_{3} \\
-y_{3} & -y_{3} & y_{2} & y_{1} \\
-y_{3} & y_{3} & y_{1} & -y_{2}
\end{array}\right], \\
& =x_{1} X_{1}+x_{2} X_{2}+x_{3} X_{3} & =y_{1} Y_{1}+y_{2} Y_{2}+y_{3} Y_{3} & \\
\mathbf{X}_{2} & =\left[\begin{array}{rrrr}
x_{1} & x_{2} & x_{3} & x_{3} \\
-x_{2} & x_{1} & x_{3} & -x_{3} \\
-x_{3} & -x_{3} & x_{1} & -x_{2} \\
-x_{3} & x_{3} & -x_{2} & x_{1}
\end{array}\right], \mathbf{Y}_{2}=\left[\begin{array}{rrrr}
y_{1} & y_{2} & y_{3} & y_{3} \\
y_{2} & -y_{1} & y_{3} & -y_{3} \\
y_{3} & y_{3} & -y_{2} & -y_{1} \\
y_{3} & -y_{3} & -y_{1} & y_{2}
\end{array}\right], \\
& =x_{1} U_{1}+x_{2} U_{2}+x_{3} U_{3}
\end{array}
$$

where $X_{1}, \ldots, V_{3}$ are the obvious $(0,1)$-matrices.

Then we have:

LemMa 16. Suppose that there is a set of pairwise amicable weighing matrices (or orthogonal designs) $\left\{M_{1}, \ldots, M_{s} ; N_{1}, \ldots, N_{t}\right\}$ of order $m$ and weights $\left(m_{1}, \ldots, m_{s}\right.$; $\left.n_{1}, \ldots, n_{\mathrm{s}}\right)$ where

$$
M_{i}^{\mathrm{T}}=-M_{i} \forall i \text { and } N_{j}^{\mathrm{T}}=N_{j} \forall j .
$$

Then there are amicable orthogonal designs of order $4 m$ and types

(i) $\left(1, m_{a}, m_{b}, 2 m_{c}\right)$ and $\left(n_{d}, m_{e}, 2 m_{t}\right)$;

(ii) $\left(1, m_{a}, n_{b}, 2 n_{c}\right)$ and $\left(n_{d}, n_{e}, 2 n_{f}\right)$;

(iii) $\left(1, m_{a}, n_{b}, 2 m_{c}\right)$ and $\left(n_{d}, n_{e}, 2 m_{f}\right)$;

(iv) $\left(1, m_{a}, m_{b}, 2 n_{c}\right)$ and $\left(n_{d}, m_{e}, 2 n_{f}\right)$;

where $m_{i} \in\left\{m_{1}, \ldots, m_{s}\right\}, i \in\{1, \ldots, s\}$ and $n_{j} \in\left\{n_{1}, \ldots, n_{3}\right\}, j \in\{1, \ldots, t\}$. 
Proof. Use the matrices defined by (2) above. The required designs are

(i)

and

(ii)

and

$$
u_{1} I \times I+u_{2} V_{1} \times M_{a}+u_{3} V_{2} \times M_{b}+u_{4} V_{3} \times M_{c}
$$

$$
v_{1} U_{1} \times N_{d}+v_{2} U_{2} \times M_{e}+v_{3} U_{3} \times M_{f} ;
$$

$$
u_{1} I \times I+u_{2} U_{1} \times M_{a}+u_{3} U_{2} \times N_{b}+u_{4} U_{3} \times N_{c}
$$

$$
v_{1} V_{1} \times N_{d}+v_{2} V_{2} \times N_{e}+v_{3} V_{3} \times N_{f}
$$

(iii)

and

$$
u_{1} I \times I+u_{2} X_{1} \times M_{a}+u_{3} X_{2} \times N_{b}+u_{4} X_{3} \times M_{c}
$$

(iv)

and

$$
\begin{gathered}
v_{1} Y_{1} \times N_{d}+v_{2} Y_{2} \times N_{e}+v_{3} Y_{3} \times M_{f} ; \\
u_{1} I \times I+u_{2} Y_{1} \times M_{a}+u_{3} Y_{2} \times M_{b}+u_{4} Y_{3} \times N_{c}
\end{gathered}
$$

$$
v_{1} X_{1} \times N_{d}+v_{2} X_{2} \times M_{e}+v_{3} X_{3} \times N_{f} ;
$$

where $u_{1}, u_{2}, u_{3}, u_{4}, v_{1}, v_{2}, v_{3}$ are commuting variables.

These give with the results quoted above and Lemma 12 of Robinson and Seberry (to appear):

LEMMA 17. In order 16 there exist best pairs $(a, b)(=$ repeat designs $(1 ;(a) ; b))$ for all $a=1,2, \ldots, 15$ and $b=1,2, \ldots, 16$ except possibly the pairs $(a, b):(13,1)$, $(13,5),(13,9),(15,7),(15,9),(15,15)$ which are undecided and $(15,1)$ which does not exist.

\section{References}

P. Delsarte, J. M. Goethals and J. J. Seidel (1971), "Orthogonal matrices with zero diagonal. II", Canad. J. Math. 23, 816-832.

A. V. Geramita, J. Murphy Geramita and J. Seberry Wallis (1975-76), "Orthogonal designs" Linear and Multilinear Algebra 3, 281-306.

A. V. Geramita and J. H. Verner (1976), "Orthogonal designs with zero diagonal", Canad. J. Math. 28, 215-224.

P. J. Robinson (1976)a, "Amicable orthogonal designs", Bull. Austral. Math. Soc. 14, 303-314.

P. J. Robinson (1976b), "A non-existence theorem for orthogonal designs", Utilitas Math. 10, 179-184.

P. J. Robinson (1977a), “Using product designs to construct orthogonal designs", Bull. Austral. Math. Soc. 16, 297-305.

P. J. Robinson (1977b), "Concerning the existence and construction of orthogonal designs", Ph.D. Thesis, Australian National University, Canberra.

P. J. Robinson and J. Seberry (to appear), "Orthogonal designs in powers of two", Ars Combinatoria.

D. Shapiro (1975-76) (private communication).

J. Seberry Wallis (1975), "Constructions for amicable orthogonal designs", Bull. Austral. Math. Soc. 12, 179-182.

J. Seberry Wallis and A. L. Whiteman (1975), "Some results on weighing matrices", Bull. Austral. Math. Soc. 12, 433-477.

W. W. Wolfe (to appear), "Rational quadratic forms and orthogonal designs', J. Number Theory. 
W. W. Wolfe (1976), 'Amicable orthogonal designs-existence”, Canad. J. Math., 28, 10061020.

W. W. Wolfe (1975), "Orthogonal designs - amicable orthogonal designs-some algebraic and combinatorial techniques”, Ph.D. Dissertation, Queen's University, Kingston, Ontario.

Institute of Advanced Studies

Department of Applied Mathematics

Australian National University The University of Sydney

Canberra 